\title{
Level Based Clustering Hierarchy Routing for Wireless Sensor Networks
}

\author{
Lei Han, Lin Qiao, Can Lv, Xuelu Yu \\ Computer science and Technology \\ Tsinghua University \\ Beijing, China \\ dengfengzaoji2007@126.com, qiaolin@tsinghua.edu.cn, lcpk0411@sina.com, yx192@163.com
}

\begin{abstract}
This paper presents a level based clustering routing scheme to solve these problems. There are many routing protocols for Wireless Sensor Network in the literature, while this paper studies the Low Energy Adaptive Clustering Hierarchy (LEACH) protocol, which is a well referred protocol for WSN, and put forward its methodology. Every node can have a level number according to their hops to the Base Station. Then the level information is used to select Cluster Head (CH) and Second Cluster Head (SCH). Like LEACH, this paper also divided into rounds to setup clusters and transmit data. But it has different two kinds of cluster heads and in each round $\mathrm{CH}$ do the different things. When $\mathrm{CH}$ is selected, it will pick out a SCH from its cluster and the SCH can substitute the $\mathrm{CH}$ when $\mathrm{CH}$ work for a stable period. This methodology improves the choice method of cluster head and the communication mode from $\mathrm{CH}$ to the Base Station.
\end{abstract}

Keywords-Wireless Sensor Networks; Level Based Routing; Clustering Hierarchy; Energy efficient

\section{INTRODUCTION}

Wireless Sensor Networks (WSN) consist of hundreds or thousands of sensor nodes powered by tiny battery. Each node has the ability of computing and communication. In WSN, Sensor nodes observe a physical phenomenal of the environment around. When observed local data, they wirelessly communicate information to an outside Base Station (BS) or sink node [7]. The BS or sink node analyzes information and makes rapid response. The advantage of WSN is that the network organization and maintenance is spontaneous and the deployment is uncomplicated. And it can work in the environment where human involvement is difficult or sometimes impossible.

Because of the limitation of power and signal strength, communication between the sensor node and the base station is costly. So the energy is the challenging subject and design factor. Many data routing algorithms are proposed to make data transfer more efficiently. In hierarchical routing $[2,3,4,5]$, sensor nodes play different roles in the network. Nodes make up many clusters and each cluster select a cluster head $(\mathrm{CH}) . \mathrm{CH}$ acts as intermediate router to the sink node or BS. Data are aggregated to $\mathrm{CHs}$ from other nodes in the cluster and $\mathrm{CHs}$ do some data fusion or compression and then send data to the BS. In order to make $\mathrm{CHs}$ live longer, nodes should become $\mathrm{CHs}$ in turn. This may achieves a large reduction in the energy consumption, as computation is much cheaper than communication [6].
In this paper, level based clustering hierarchy routing methodology is proposed. Every node can have a level number according to their hops to the Base Station. Then the level information is used to select Cluster Head and Second Cluster Head. The different two kinds of $\mathrm{CHs}$ do the different things. When the $\mathrm{CH}$ is selected, it will pick out a $\mathrm{SCH}$ from its cluster and the $\mathrm{SCH}$ can substitute the $\mathrm{CH}$ when $\mathrm{CH}$ work for a stable period. This methodology improves the choice method of cluster head and the communication mode from $\mathrm{CH}$ to the Base Station.

The reset of this paper organized as follows. Section II describes some closely related work on WSN routing. Section III represents the network model. Section IV describes proposed work in detail. Some concluding remarks are given in Section V.

\section{RELATED WORK}

Many research work has been performed on routing algorithms for wireless sensor networks. In general, routing problem has received a great interest from the research community. Low Energy Adaptive Clustering Hierarchy (LEACH) [2] is a cross layered routing protocol which combines medium routing access to collect and transmit data to Base Station (BS). The good performance of LEACH is increasing the network lifetime and decreasing the network energy consumption. LEACH uses hierarchical approach and separates the network into a set of clusters. Each cluster has a selected cluster head $(\mathrm{CH})$ and the $\mathrm{CH}$ administrate other nodes in the cluster. $\mathrm{CH}$ aggregates the data from other nodes and transmits the collected data directly to BS.

The function to select the $\mathrm{CH}$ of $\mathrm{LEACH}$ is divided into rounds that organized in two phases. The setup phase starts with the self-election of nodes to become $\mathrm{CHs}$ and ensures $\mathrm{CH}$ role rotates among nodes to balance the energy consumption while the steady-state phase is aimed at the selected $\mathrm{CH}$ does data collection, aggregation, and transmit to BS. LEACH makes the sensors within their cluster dissipate their energy slowly, while the $\mathrm{CHs}$ that located farther away from the BS may consume more energy. Also, LEACH does not guarantee good $\mathrm{CH}$ distribution.

Some protocols further improved LEACH in many aspects. LAECH-Centralized (LEACH-C) [3] uses a centralized clustering algorithm and same steady-state protocol. Each node sends their location and energy information to the BS. Then BS will determine clusters and $\mathrm{CH}$ node of each cluster. The BS know the global 
information of the network so that it can produce better clusters that require less energy for data transmission. What's more, the number of $\mathrm{CHs}$ in each round equals a predetermined optimal value.

Enhanced LEACH (E-LEACH) [3] improved in two major aspects. One is that the cluster head selection algorithm assumes that sensors have global information about other sensors' remaining energy. The other is the required number of cluster heads has to scale as the square root of the total number of sensor nodes to minimize the total energy consumption under certain assumptions.

Multi-hop LEACH (M-LEACH) [4] allow sensor nodes to use multi-hop communication within the cluster in order to increase the energy efficiency. The main innovation of the solution proposed here is that the multi-hop approach is followed inside the cluster and outside the cluster.

LEACH with Fixed Cluster (LEACH-F) [5] is based on clusters that are formed once and then fixed. The $\mathrm{CH}$ position rotates among the nodes within the cluster. Once the clusters are formed, there is no set-up overhead at the beginning of each round. But it does not allow new nodes to be added to the network or adjust the behavior based on nodes dying.

The author [1] proposed Level Based Flooding (LBF). In $\mathrm{LBF}$, the network is divided into several layers. The sink node knows each node's level information. The search packet is broadcast according to levels of nodes to the target node. When target node receives the packet, it sends the data back to the sink node in random walk within level hops.

The author [8] proposed Level and Cluster Based Routing. The nodes have the same level number are split into the same cluster. When the cluster head is chosen, it will never change in its cluster. The cluster head manage other nodes to work in turn. And the author [9] proposed an adaptive and energy efficient routing which focus on the QOS of the network.

\section{NETWORK MODEL}

In this paper, the network model is considered as followed. It is assumed that all sensor nodes in the network are homogeneous and static, and they are uniformly deployed. Each node has a transmission range of a radius $\mathrm{R}$ circle and all nodes can get its message in the transmission range. What's more, all nodes can control their transmission range by adjusting transmission power. Base Station is located inside the sensor filed and all nodes can directly communication with it.

\section{ALGORITHM DESIGN}

Our proposed scheme composed of two phases:

A. Route Construction Phase, and

B. Data Forwarding Phase.

\section{A. Route Construction Phase}

Route construction phase can be divided into two steps. Level Determination Step and Cluster Establishment Step. The Level Determination Step is similar to LBF [1].

1) Level Determination Step

In this step, base station start the level determination process. The BS broadcast a packet by adding its level information to it. At the beginning, the level of the BS is considered to be zero. When a node receives a packet, if the packet's level is smaller than the node's level, it will update its value as the packet's level value plus one. Then it rebroadcast the packet by updating the packet's level with its level. Otherwise it will drop the packet. Each node is also collects its neighbor's level information. Then nodes can segregate their neighbors into two sets as follow: LowLevelNeighbors(LLN): Set of all neighbors whose level is smaller than that of the node; NearLevelNeighbors(NLN): Set of all neighbors whose level is equal to or just differ only one level that of the node.

\section{2) Cluster Establishment Step}

After Level Determination Step every node has a level number, so WSN goes to Cluster Establishment Step. The operation of Cluster Establishment Step is broken up into rounds, where each round starts with a set-up phase to organize the clusters and followed by a steady-state phase to transfer data to the base station. In order to minimize overhead, the steady-state is long compared to the set-up phase.

Each node decides whether or not to become a cluster head $(\mathrm{CH})$ for the current round when clusters are being created. In order to increase lifetime of the network, node having more residual energy than others should have high probability to become $\mathrm{CH}$. It is proposed to use the energy current rate $E_{c t}=E_{\text {residual }} / E_{\text {init }}$ of node for selection of $\mathrm{CH}$. The distance between $\mathrm{CH}$ and $\mathrm{BS}$ is directly proportional of the energy consumption in data transmission. And it is proposed to consider the node's level number $\mathrm{L}_{\mathrm{p}}=\mathrm{L}_{\mathrm{n}} /\left(\mathrm{L}_{\mathrm{max}}+1\right)$ for selection of $\mathrm{CH}$.

The node $\mathrm{n}$ generates a random number between 0 and 1 , if the number is less than a threshold $\mathrm{T}(\mathrm{n})$, the node becomes a $\mathrm{CH}$ for the current round. The threshold of a contending node $\mathrm{n}$ is articulated as follows:

$$
T(n)= \begin{cases}P /[1-P \times(r \bmod [1 / p])] \times\left[\left(1-L_{p}\right) \times E_{c t}+4 L_{p}{ }^{2}-4 L_{p}{ }^{3}\right], \text { if } n \in G \\ 0 & \text { otherwise }\end{cases}
$$

Where $\mathrm{P}$ is the desired percentage of $\mathrm{CH}, \mathrm{r}$ is the current round, $\mathrm{G}$ is set of nodes that has not been $\mathrm{CH}$ in past $1 / \mathrm{P}$ rounds, Ecurrent is the current energy, Einit is the initial energy, Ln is the level number and Lmax is the max level number of all sensor nodes.If node lies near the BS then energy is given more weight age compared to level number for $\mathrm{CH}$ selection. If node lies far away from the BS then level number is given more weight age compared to energy for $\mathrm{CH}$ selection. If node lies near the middle position then nearly equal weight age is given to energy and level number of node. So the expression $L_{p} \times\left[1-\left(2 L_{p}-1\right)^{2}\right]=4 L_{p}{ }^{2}-4 L_{p}{ }^{3}$ is used.

Then $\mathrm{CH}$ will select a second cluster head $(\mathrm{SCH})$ from its NearLevelNeighbors(NLN). CH broadcasts an advertisement message to neighbors. And all $\mathrm{CHs}$ transmit their advertisement using the same transmit energy. When a sensor node receives an advertisement from a $\mathrm{CH}$, if it is a $\mathrm{CH}$, it drops the message. If it hasn't joint in a cluster, it will join in this cluster and remember the $\mathrm{CH}$ id as well as save 
calculated signal strength. If old CH's signal strength is weaker than the new one, it will join in the new cluster and save the new CH's information.

After this phase is complete, each sensor node decides the cluster to which it will belong for this round. Then these nodes send a package to their $\mathrm{CH}$ including current energy and level number. $\mathrm{CH}$ stores sensor nodes within its cluster in local cache and select the node that has the most remaining energy whose level number is the closest to $\mathrm{CH}$ to be second cluster head ( $\mathrm{SCH})$. After that, $\mathrm{CH}$ broadcasts a message to its cluster members, telling them the SCH's id. When SCH knows its identity, it goes into sleep until next phase is coming.

After cluster is built, it goes to the steady-state. This state is average divided into two parts. The first part is for the selected $\mathrm{CH}$ and the second part is for the selected $\mathrm{SCH}$ does data collection, aggregation and delivery to BS. Between the first part and the second part, there's a behavior of changing the head node. The $\mathrm{CH}$ goes to sleep while the $\mathrm{SCH}$ stop sleeping and becomes a $\mathrm{CH}$. All the sensor nodes are in the same cluster and they know the new CH's information, so it can save a lot of consumption from communication. The function of my method is shown in Fig. 1, the algorithm of cluster establishment step is shown in Fig. 2 and the flow chart of cluster establishment step is shown in Fig. 3.

\section{B. Data Forwarding Phase}

Although the $\mathrm{CH}$ can transmit directly to the $\mathrm{BS}$, it will cost a lot of energy if the $\mathrm{CH}$ is far away from the BS. So multi-hop routing is considered instead of single-hop routing. When $\mathrm{CH}$ has collected data from the sensor nodes in its cluster, it could create a multi-hop path to forward data to the BS. As ever node has a level number, data will transmit towards level smaller direction. The residual energy is also considered at each $\mathrm{CH}$. When the $\mathrm{CH}$ picks up a next-hop node, it first checks the current energy of that node. The $\mathrm{CH}$ selects a path with the next-hop node whose current energy higher than a threshold energy value and level number is smaller than the $\mathrm{CH}$.

If the $\mathrm{CH}$ has several choices about the next-hop node, it then selects one of them whose level number is the biggest among them. And if more than one node has the same biggest level number, the $\mathrm{CH}$ selects the more residual energy one. Each $\mathrm{CH}$ performs the periodic exchange of asking packet to inform its lower level number neighbor $\mathrm{CHs}$

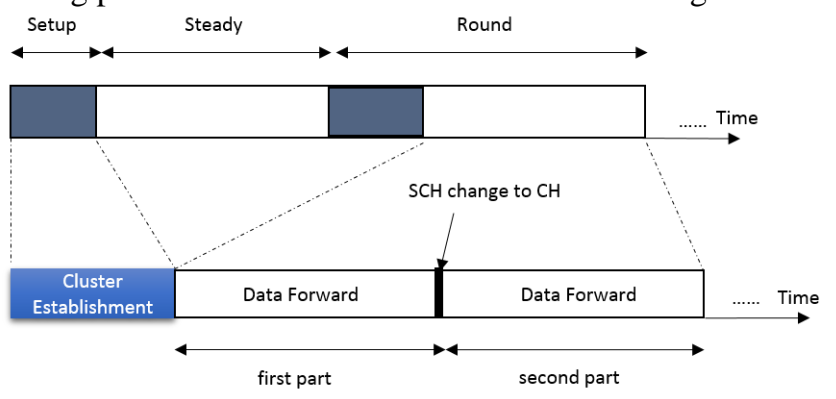

Fig.1 Time line showing the operation of my method of its current energy. If a CH's residual energy goes to lower than the threshold energy then it cannot take part in as a router in forwarding data over a path. It just acts as a normal $\mathrm{CH}$ in the environment and can send only data of itself to others. If a $\mathrm{CH}$ realizes that all its neighbor $\mathrm{CHs}$ lack their residual energy below the threshold energy that cannot act as a router in forwarding data, the $\mathrm{CH}$ transmit the collected data directly to the $\mathrm{BS}$.

\section{Load Balance Schedule}

A feature of Wireless Sensor Networks is redundant deployment. Many sensor nodes are deployed in the same range of the network. They may get the same environment events when they sense the same area. In this paper's schema sensors in the same cluster detect the same area, so it is a waste of resource if all the sensor nodes work all the time. A strategy is proposed to schedule them to work or sleep. In this paper's schema, CHs and SCHs take the scheduling job duty. They know the information of energy and level number of other nodes in their cluster. $\mathrm{CH}$ choose $\mathrm{N}$ (calculated through experiments) number sensor nodes in its cluster to

\section{Begin}

1. Node Ni calculate its threshold $\mathrm{T}(\mathrm{Ni})$ by its energy and level number.

2. Ni generates a random number Ri between 0 and 1 .

$$
\text { 3. If } \mathrm{Ri}<\mathrm{T}(\mathrm{Ni})
$$

$$
\text { Else }
$$

Goto Step 4

Goto Step 6.

4. Ni turns into a cluster head and broadcast its cluster head state to neighbors.

5. Ni wait request from other nodes.

Select the second cluster head and send the information to the nodes in its cluster.

Work as a cluster head until the cluster head change phase. Goto Step 10.

6. Ni wait information from cluster head

7. Ni send information of its energy and level number to its cluster head

8. Ni wait information from its cluster head about the second cluster head

If it is the second cluster head

Goto Step 9.

$$
\text { Else }
$$

Goto Step 12.

9. Ni goes to sleep until the cluster head change phase. Goto Step 12.

10. If cluster head change phase is coming Ni goes to sleep. Goto Step12.

11. If cluster head change phase is coming

Ni turns into a cluster head. Goto Step12.

12. Cluster formed.

End

Fig.2 Algorithm of cluster establishment step 
work according to their energy left and level number while the left idle nodes go to sleep for a fixed time. When $\mathrm{SCH}$ replace the $\mathrm{CH}$ role, it will be in charge of the scheduling work. This method can improve the energy efficient of the network.

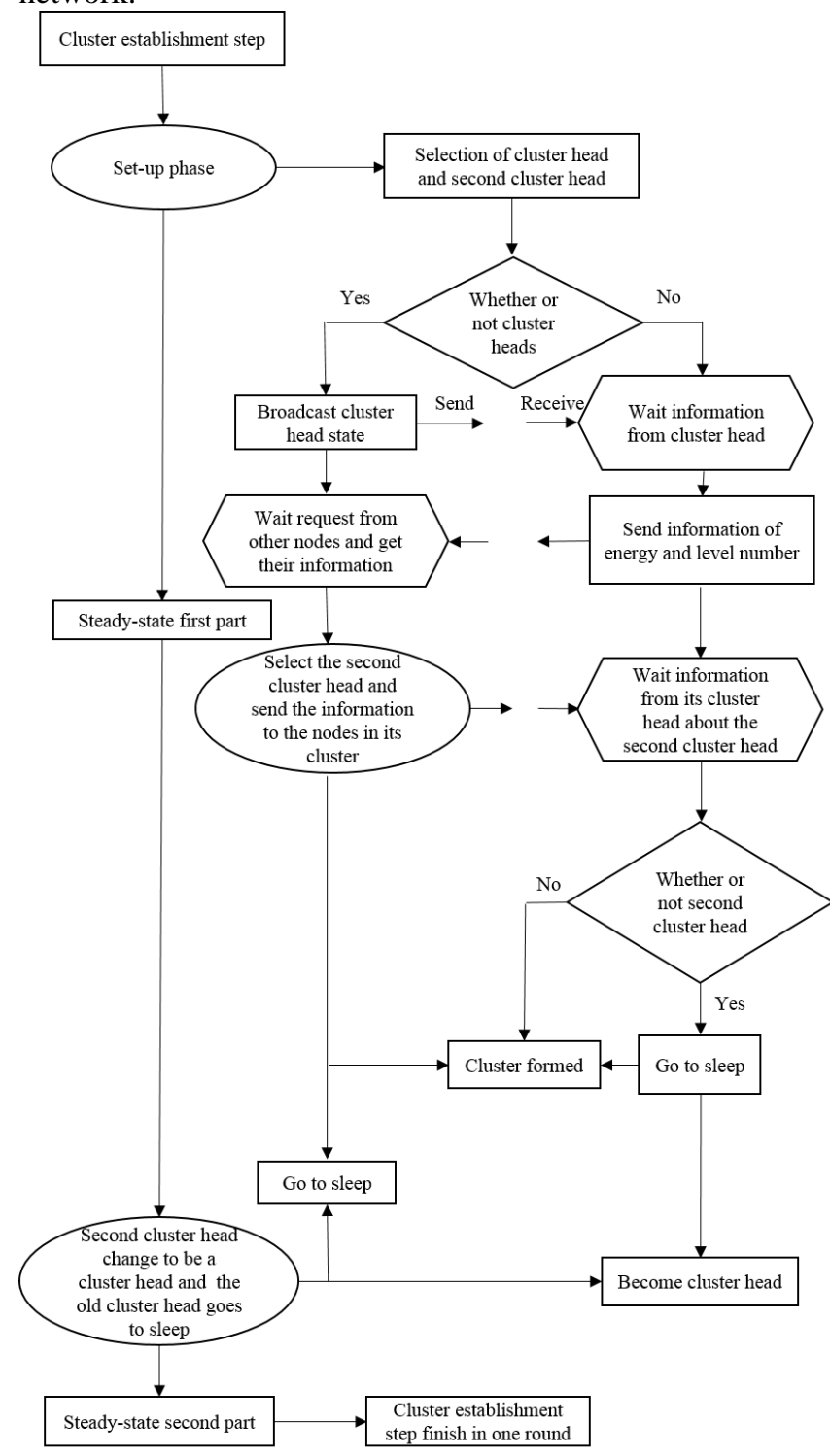

Fig.3 Flow chart of cluster establishment step

\section{CONCLUSION}

In this paper, A level based clustering hierarchy routing methodology is proposed. The most important factor is the energy efficient. In this paper, sensor nodes in the network get their level number according to their hops to the BS. And nodes are split into different clusters. Using the level information it can choose a multi-hop path to transfer data from $\mathrm{CH}$ to $\mathrm{BS}$. $\mathrm{CH}$ and $\mathrm{SCH}$ are scheduled to work in turn according to the level number and residual energy. This makes energy consumption of whole work more balance. The further jobs will focus on the following directions: (1) evaluating our level based clustering hierarchy routing methodology through simulation, and (2) making the time of alternately of $\mathrm{CH}$ and $\mathrm{SCH}$ can change according to their energy left.

\section{REFERENCES}

[1] Yanhong Ding, Tie Qiu, Honglian Ma, and Naigao Jin: "Level Based Flooding for Node Search in Wireless Sensor Network". Proc. of the 2011 2nd International Congress CACS, AISC 145, pp.483,488, 2012.

[2] Heinzelman, W.R.; Chandrakasan, A.; Balakrishnan, H., "Energyefficient communication protocol for wireless microsensor networks," System Sciences, 2000. Proceedings of the 33rd Annual Hawaii International Conference on, vol., no., pp.10 pp. vol.2,, 4-7 Jan. 2000.

[3] Heinzelman, W.B.; Chandrakasan, A.P.; Balakrishnan, H., "An application-specific protocol architecture for wireless microsensor networks," Wireless Communications, IEEE Transactions on, vol.1, no.4, pp.660,670, Oct 2002.

[4] Mhatre, V.; Rosenberg, C., "Homogeneous vs heterogeneous clustered sensor networks: a comparative study," Communications, 2004 IEEE International Conference on, vol.6, no., pp.3646,3651 Vol.6, 20-24 June 2004

[5] W.R. Heinzelman, ."Application-Specific Protocol Architecture for Wireless Networks". PhD Thesis, Massachusetts Institute of Technology, June 2000.

[6] Ameer Ahmed Abbasi, Mohamed Younis: "A survey on clustering algorithms for wireless sensor networks," Computer Communications 30 pp. 2826,2841, 2007.

[7] Misra, S., "Reviews of "multi-gigabit microwave and milimeter-wave wireless communication" (wells, j.; 2010) and "wireless sensor networks: a networking perspective" (zheng, j. and jamalipour, a.; 2009) [book reviews]," Wireless Communications, IEEE, vol.19, no.3, pp.10,11, June 2012

[8] Koulin Yuan, Lin Qiao, LeiHan: "Level and Cluster Based Routing for Wireless Sensor Network," Applied Mechanics and Materials, Volumes 321,324, pp.515,522, June 2013.

[9] Koulin Yuan, Lin Qiao, Lei Han, Zhizhong Tang: "A Self-Adaptive and Energy Efficient Routing Approach for Wireless Sensor Network," In: Proceedings of 2013 Fourth International Conference on Digital Manufacturing and Automation (ICDMA 2013), pp.73,76, June 2013. 